\title{
Brane-world black holes and energy-momentum vector
}

\section{Mustafa Saltı and Oktay Aydoğdu}

Department of Physics, Art and Science Faculty, Middle East Technical University 06531, Ankara-Turkey

E-mail: musts6@yahoo.com, oktay231@yahoo.com

\section{Murat Korunur}

Department of Physics, Faculty of Art and Science, Dicle University

21280, Diyarbakir-Turkey

E-mail: mkorunur@dicle.edu.tr

ABSTRACT: The Brane-World black hole models are investigated to evaluate their relative energy and momentum components. We consider Einstein and Møller's energy-momentum prescriptions in general relativity, and also perform the calculation of energy-momentum density in Møller's tetrad theory of gravity. For the Brane-World black holes we show that although Einstein and Møller complexes, in general relativity give different energy relations, they yield the same results for the momentum components. In addition, we also make the calculation of the energy-momentum distribution in teleparallel gravity, and calculate exactly the same energy as that obtained by using Møller's energy-momentum prescription in general relativity. This interesting result supports the viewpoint of Lessner that the Møller energy-momentum complex is a powerful concept for the energy and momentum. We also give five different examples of Brane-World black holes and find the energy distributions associated with them. The result calculated in teleparallel gravity is also independent of the teleparallel dimensionless coupling constant, which means that it is valid in any teleparallel model. This study also sustains the importance of the energy-momentum definitions in the evaluation of the energy distribution of a given space-time, and supports the hypothesis by Cooperstock that the energy is confined to the region of non-vanishing energy-momentum tensor of matter and all non-gravitational fields.

KeYwords: Classical Theories of Gravity, Black Holes, Black Holes in String Theory. 


\section{Contents}

1. Introduction 1

2. Energy-momentum distribution in general relativity 3

2.1 Einstein's 4-momentum formulation

2.2 Møller's 4-momentum definiton

3. Relative energy in teleparallel gravity 5

4. Examples 8

5. Discussions 11

\section{Introduction}

Randall and Sundrum [1, 2] introduced a model that captures some of the essential features of the dimensional reduction of eleven-dimensional super-gravity proposed by Hor̃ava and Witten [3, [4. The second Randall-Sundrum scenario [2] is a five dimensional anti-de Sitter bulk spacetime with an embedded Minkowski 3-brane where matter fields are confined and Newtonian gravity is effectively re-produced at low energies [5]. The second RandallSundrum scenario was generalized to a Friedmann-Robertson-Walker brane, shoving that the Friedmann equation at high energies gives $H^{2} \sim \rho^{2}$, in contrast with the general relativistic behavior $\left.H^{2} \sim \rho[6]-8\right]$.

The gravitational field on the brane is defined by the modified Einstein equations obtained by Shiromizu, Maeda and Sasaki [9] from five-dimensional gravity with the help of the Gauss and Codazzi equations [10]:

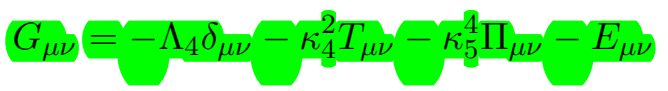

where

$$
G_{\mu \nu}=R_{\mu \nu}-\frac{1}{2} \delta_{\mu \nu} R
$$

is the four-dimensional Einstein tensor, $\Lambda_{4}$ is the four-dimensional cosmological constant expressed in terms of the five-dimensional cosmological constant $\Lambda_{5}$ and the brane tension $\varrho$

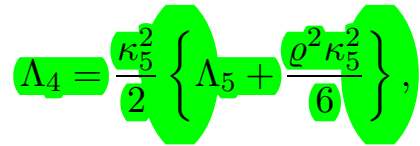

Here, $\kappa_{4}^{2}=8 \pi G_{N}=\varrho \kappa_{5}^{2} / 6 \pi$ is the four-dimensional gravitational constant (here $G_{N}$ is Newton's constant of gravity); $T_{\mu \nu}$ is the stress energy tensor of the matter confined on 
the brane; $\Pi_{\mu \nu}$ is a tensor quadratic in $T_{\mu \nu}$, obtained from matching the five-dimensional metric across the brane

$$
2 \Pi_{\mu}^{\nu}=T_{\mu}^{\beta} T_{\beta}^{\nu}-T T_{\mu}^{\nu}-\delta_{\mu}^{\nu}\left(T_{\gamma \rho} T^{\gamma \rho}-\frac{T^{2}}{2}\right)
$$

where $T=T_{\mu}^{\mu}$; and $E_{\mu \nu}$ is the electric part of the five-dimensional Weyl tensor projected onto the brane: in proper five-dimensional coordinates, $E_{\mu \nu}=\delta_{\mu}^{M} \delta_{\nu}^{N} C_{M N K L} n^{K} n^{L}$ where $M, N, \ldots$ are five-dimensional indices and $n^{M}$ is the unit normal to the brane [11]. Our interest here is in selecting a general class of static, spherically symmetric black holes to equation (1.1) without specifying $E_{\mu \nu}$.

The general static, spherically symmetric four-dimensional line-element in the curvature coordinates has the following form,

$$
d s^{2}=\Im(r) d t^{2}-\frac{r}{\Xi(r)} d r^{2}-r^{2}\left(d \theta^{2}+\sin ^{2} \theta d \phi^{2}\right) .
$$

In particular examples we mostly deal with asymptotically flat vacuum solutions, such that $\Lambda_{4}=T_{\mu}^{\nu}=0$. If we treat equation (1.1) as the conventional Einstein equations with an effective stress energy tensor $\widehat{T}_{\mu \nu}$ (the hat means effective); i.e.

$$
\widehat{G}_{\mu \nu}=-\kappa_{4}^{2} \widehat{T}_{\mu \nu}
$$

then we get

$$
\begin{aligned}
\widehat{T}_{0}^{0}= & \widehat{\rho}=\frac{1}{\kappa_{4}^{2} r^{3}}\left[1-r \partial_{r} \Xi(r)\right], \\
\widehat{T}_{1}^{1}= & \widehat{\rho}_{\mathrm{rad}}=\frac{1}{\kappa_{4} r^{2}}\left[\frac{1-\Xi(r)}{r}+\frac{\Xi(r) \partial_{r} \Im(r)}{\Im(r)}\right], \\
\widehat{T}_{2}^{2}= & \widehat{T}_{3}^{3}=\widehat{\rho}_{\operatorname{tang}}=\frac{1}{\kappa_{4}^{2}}\left[\frac{2 \partial_{r}^{2} \Im(r)}{\Im(r)}-\frac{\left[\partial_{r} \Im(r)\right]^{2}}{\Im(r)}+\frac{\partial_{r} \Im(r)\left[r \partial_{r} \Xi(r)-\Xi(r)\right]}{r \Im(r) \Xi(r)}\right. \\
& \left.+\frac{2}{r^{2}}\left(\partial_{r} \Im(r)-\frac{\partial_{r} \Xi(r)-\Xi(r)}{r \Xi(r)}\right)\right]
\end{aligned}
$$

Hence, it is very interesting to discuss energy associated with this general brane-world black hole model. In this study to calculate energy in this brane-world black hole solution, we focus on the Møller and Einstein energy-momentum formulations in general relativity and also on Møller's formulation in teleparallel gravity.

Since Einstein introduced general relativity, relativists have not been able to agree upon a definition of the energy-momentum distribution associated with the gravitational field [12]. Einstein [13] first obtained such an expression and many others such as LandauLifshitz, Papapetrou, Weinberg, Bergmann-Thomson, Tolman, Møller and Qadir-Sharif gave similar prescriptions [14]. The expressions they gave are called energy-momentum complexes because they can be expressed as a combination of energy-momentum density which is usually defined by a second rank tensor $T_{i}^{k}$ and a pseudo-tensor, which is interpreted to represent the energy and momentum of the gravitational field. These formulations have been heavily criticized because they are non-tensorial, i.e. they are coordinate 
dependent. Except for the Møller definition these formulations only give meaningful results if the calculations are performed in Cartesian coordinates. Møller proposed a new expression for the energy-momentum complex which could be utilized to any coordinate system. Next, Lessner [15] argued that the Møller prescription is a powerful concept for energy-momentum in general relativity.

Recently, the problem of energy-momentum localization has also been considered in teleparallel gravity [16, 17]. Møller showed that a tetrad description of a gravitational field equation allows a more satisfactory treatment of the energy-momentum complex than does general relativity. Therefore, we have also applied the super-potential method by Mikhail et. al. [18] to calculate the energy of the central gravitating body. Vargas [16], using the definitions of Einstein and Landau-Lifshitz in teleparallel gravity, found that the total energy is zero in Friedmann-Robertson-Walker space-times. After this work there are a few papers on the energy-momentum in teleparallel gravity [19-21].

Considerable efforts have also been performed in constructing super-energy tensors [22]. Motivated by the works of Bel [23] and independently of Robinson [24], many investigations have been carried out in this field [25].

The paper is organized as follows. In the next section 2, we calculate energy in general relativity by using Einstein and Møller's energy-momentum formulations. Section 3 gives us the energy of a given model in Møller's tetrad theory of gravity. Next, in section 4 , we give some exeamples. Finally, last section 5 is devoted to discussions. In this paper we use convention that $G=1$ and $c=1$. Except for the cases, we give the special values of the indices, all indices take the values from 0 to 3 .

\section{Energy-momentum distribution in general relativity}

Virbhadra and collaborators revived the interest in this approach [26, 27] and since then numerous works on evaluating the energy and momentum distributions of several gravitational backgrounds have been completed [28-32]. Later attempts to deal with this problematic issue were made by proposers of quasi-local approach. The determination as well as the computation of the quasi-local energy and quasi-local angular momentum of a (2+1)-dimensional gravitational background were first presented by Brown, Creighton and Mann [33]. A large number of attempts since then have been performed to give new definitions of quasi-local energy in Einstein's theory of general relativity [34]. Furthermore, according to the Cooperstock hypothesis [35], the energy is confined to the region of non-vanishing energy-momentum tensor of matter and all non-gravitational fields.

\subsection{Einstein's 4-momentum formulation}

The formulation of the Einstein prescription [13] is defined as

$$
\wp_{\mu}^{\nu}=\frac{1}{16 \pi} \triangle_{\mu, \alpha}^{\nu \alpha}
$$

where

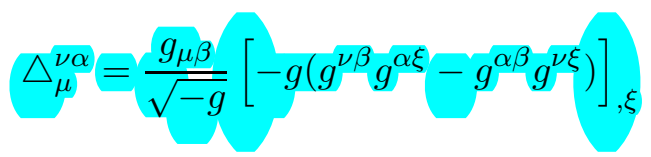


$\wp_{0}^{0}$ is the energy density, $\wp_{k}^{0}$ (where $\left.k=1,2,3\right)$ are the momentum density components, and $\wp_{0}^{k}$ are the components of energy-current density. The Einstein energy and momentum density satisfies the local conservation laws

$$
\frac{\partial \wp_{\mu}^{\nu}}{\partial x^{\nu}}=0
$$

and the energy-momentum components are given by

$$
P_{\mu}=\iiint \wp_{\mu}^{0} d x d y d z \quad(\mu=0,1,2,3) .
$$

$P_{\mu}$ is called the momentum four-vector; $P_{k}$ (here $\left.k=1,2,3\right)$ give momentum components $P_{1}$, $P_{2}, P_{3}$ and $P_{0}$ gives the energy.

In order to use the Einstein energy-momentum complex, we have to transform the line element (1.5) in quasi-Cartesian coordinates. According to

$$
\begin{aligned}
& x=r \sin \theta \cos \phi, \\
& y=r \sin \theta \sin \phi, \\
& z=r \cos \theta,
\end{aligned}
$$

one gets

$$
d s^{2}=\Im(r) d t^{2}-\left(d x^{2}+d y^{2}+d z^{2}\right)-\frac{r-\Xi(r)}{r^{2} \Xi(r)}(x d x+y d y+z d z)^{2} .
$$

Using the metric transformed into quasi-Cartesian coordinates with equations (2.1), (2.2) and (2.4), one gets the following expression for the energy distribution

$$
E_{\text {Einstein }}^{G R}(r)=\frac{1}{2} \sqrt{\frac{r \Im}{\Xi}}(r-\Xi),
$$

and for the momentum components, we have

$$
\vec{P}_{\text {Einstein }}^{G R}(r)=0 \text {. }
$$

\subsection{Møller's 4-momentum definiton}

In general relativity, Møller's energy-momentum complex is given by [14

$$
\Upsilon_{\mu}^{\nu}=\frac{1}{8 \pi} \frac{\partial \Theta_{\mu}^{\nu \alpha}}{\partial x^{\alpha}}
$$

satisfying the local conservation laws:

$$
\frac{\partial \Upsilon_{\mu}^{\nu}}{\partial x^{\nu}}=0
$$

where the antisymmetric super-potential $\Theta_{\mu}^{\nu \alpha}$ is

$$
\Theta_{\mu}^{\nu \alpha}=\sqrt{-g}\left\{\frac{\partial g_{\mu \beta}}{\partial x^{\gamma}}-\frac{\partial g_{\mu \gamma}}{\partial x^{\beta}}\right\} g^{\nu \gamma} g^{\alpha \beta}
$$


The locally conserved energy-momentum complex $\Upsilon_{\mu}^{\nu}$ (here $\left.\mu, \nu=0,1,2,3\right)$ contains contributions from the matter, non-gravitational and gravitational fields. $\Upsilon_{0}^{0}$ is the energy density and $\Upsilon_{i}^{0}$ (here $i=1,2,3$ ) are the momentum density components. The momentum four-vector definition of Møller is given by

$$
P_{\mu}=\iiint \Upsilon_{\mu}^{0} d x d y d z \quad(\mu=0,1,2,3) .
$$

Using Gauss's theorem, this definition transforms into

$$
P_{\mu}=\frac{1}{8 \pi} \iint \Theta_{\mu}^{0 i} \zeta_{i} d S
$$

where $\zeta_{i}$ (where $i=1,2,3$ ) is the outward unit normal vector over the infinitesimal surface element $d S$. $P_{i}$ give momentum components $P_{1}, P_{2}, P_{3}$ and $P_{0}$ gives the energy.

Next, using equation (2.13) with the metric (1.5), we obtain the following non-zero component of the antisymmetric super-potential $\Theta_{\mu}^{\nu \alpha}$

$$
\Theta_{0}^{01}(r, \theta)=\frac{r^{2} \Im^{\prime}(r) \sin \theta}{\sqrt{\frac{r \Im(r)}{\Xi(r)}}},
$$

while the momentum density distributions take the form

$$
\begin{aligned}
& \Upsilon_{1}^{0}=0 \\
& \Upsilon_{2}^{0}=0 \\
& \Upsilon_{3}^{0}=0
\end{aligned}
$$

Hence, the Møller energy distribution is

$$
E(r)=\frac{r^{3 / 2}}{2} \Im^{\prime}(r) \sqrt{\frac{\Xi(r)}{\Im(r)}} .
$$

which is also the energy (mass) of the gravitational field that a neutral particle experiences at a finite distance $r$. Additionally, we can find the momentum components as following

$$
P_{1}^{(\text {Moller })}=P_{2}^{(\text {Moller })}=P_{3}^{(\text {Moller })}=0 .
$$

\section{Relative energy in teleparallel gravity}

Teleparallel theories, whose basic entities are tetrad fields $h_{a \mu}(a$ and $\mu$ are $\mathrm{SO}(3,1)$ and space-time indices, respectively) have been considered a long time ago by Møller 36] in connection with attempts to define the energy of the gravitational field. Teleparallel theories of gravity are defined on Weitzenböck space-time [37], which is endowed with the affine connection $\Gamma_{\mu \nu}^{\lambda}=h^{a \lambda} \partial_{\mu} h_{a \nu}$ and where the curvature tensor, constructed out of this connection, vanishes identically. This connection defines a space-time with an absolute parallelism or teleparallelism of vector fields [38]. In this geometrical framework the gravitational effects are due to the space-time torsion corresponding to the above mentioned 
connection. As remarked by Hehl [39], by considering Einstein's general relativity as the best available alternative theory of gravity, its teleparallel equivalent is the next best one. Therefore it is interesting to perform studies of the spacetime structure as described by the teleparallel gravity.

The super-potential of Møller's in teleparallel gravity is given by Mikhail et al. [18] as

$$
\begin{aligned}
M_{\mu}^{\nu \beta}= & \frac{(-g)^{1 / 2}}{2 \kappa}\left(\delta_{\chi}^{\tau}\left[\delta_{\rho}^{\nu} \delta_{\sigma}^{\beta}-\delta_{\sigma}^{\nu} \delta_{\rho}^{\beta}\right]+\delta_{\rho}^{\tau}\left[\delta_{\sigma}^{\nu} \delta_{\chi}^{\beta}-\delta_{\chi}^{\nu} \delta_{\sigma}^{\beta}\right]\right. \\
& \left.-\delta_{\sigma}^{\tau}\left[\delta_{\chi}^{\nu} \delta_{\rho}^{\beta}-\delta_{\rho}^{\nu} \delta_{\chi}^{\beta}\right]\right)\left\{\Phi^{\rho} g^{\sigma \chi} g_{\mu \tau}-\lambda g_{\tau \mu} \xi^{\chi \rho \sigma}-(1-2 \lambda) g_{\tau \mu} \xi^{\sigma \rho \chi}\right\}
\end{aligned}
$$

here $\xi_{\alpha \beta \mu}$ is the con-torsion tensor given by

$$
\xi_{\alpha \beta \mu}=h_{i \alpha} h_{\beta ; \mu}^{i}
$$

where the semicolon denotes covariant differentiation with respect to Christoffel symbols

$$
\left\{\begin{array}{c}
\alpha \\
\mu \nu
\end{array}\right\}=\frac{1}{2} g^{\alpha \beta}\left(\partial_{\mu} g_{\beta \nu}+\partial_{\nu} g_{\beta \mu}-\partial_{\beta} g_{\mu \nu}\right),
$$

and $\Phi_{\mu}$ is the basic vector field defined by

$$
\Phi_{\mu}=\xi_{\mu \rho}^{\rho},
$$

$\kappa$ is the Einstein constant, and $\lambda$ is a free dimensionless parameter.

In a space-time with absolute parallelism the teleparallel vector fields $h_{i}{ }^{\mu}$ define the non-symmetric connection

$$
\Gamma_{(\mu \beta}^{\alpha}=h_{i}^{\alpha} \partial_{\beta} h_{\mu}^{i} .
$$

The curvature tensor which is defined by $\Gamma_{\mu \beta}^{\alpha}$ is identically vanishing. Møller constructed a gravitational theory based on this space-time. In this gravitation theory the field variables are the 16 tetrad components $h_{i}^{\mu}$, from which the metric tensor is defined by

$$
g^{\alpha \beta}=h_{i}^{\alpha} h_{j}^{\beta} \eta^{i j} \text {. }
$$

We assume an imaginary value for the vector $h_{0}^{\mu}$ in order to have a Lorentz signature. We note that, associated with any tetrad field $h_{i}^{\mu}$, there is a metric field defined uniquely by equation (3.6), while a given metric $g^{\alpha \beta}$ doesn't determine the tetrad field completely; for any local Lorentz transformation of the tetrads $h_{i}{ }^{\mu}$ leads to a new set of tetrads which also satisfy equation (3.6).

The energy-momentum density is given by 36]

$$
\Xi_{\alpha}^{\beta}=M_{\alpha, \lambda}^{\beta \lambda}
$$

where comma denotes ordinary differentiation. The energy distribution $E$ and momentum components $P_{i}$ are expressed by the volume integral [36],

$$
\begin{aligned}
& \text { Moller } E^{T P}=\lim _{r \rightarrow \infty} \int_{r=\text { constant }} \Xi_{0}^{0} d x d y d z, \\
& \text { Moller } P_{i}^{T P}=\lim _{r \rightarrow \infty} \int_{r=\text { constant }} \Xi_{i}^{0} d x d y d z .
\end{aligned}
$$


Here, the index of $i$ takes the value from 1 to 3 and TP means Teleparallel Gravity. The angular momentum $J_{i}$ of a general relativistic system is given by [36]

$$
J_{i}=\lim _{r \rightarrow \infty} \int_{r=\text { constant }}\left(x_{j} \Xi_{k}^{0}-x_{k} \Xi_{j}^{0}\right) d x d y d z
$$

where $i, j$ and $k$ take cyclic values 1,2 and 3 . We are interested in determining the total energy, and the momentum components.

In the Cartesian coordinates, the general form of the tetrad $h_{i}^{\mu}$ which has spherical symmetry, is given [40] as

$$
\begin{aligned}
& h_{0}^{0}=i G_{1}, \quad h_{a}^{0}=G_{2} x^{a}, \quad h_{0}^{\alpha}=i G_{3} x^{\alpha}, \\
& h_{a}^{\alpha}=G_{4} \delta_{a}^{\alpha}+G_{5} x^{a} x^{\alpha}+\epsilon_{a \alpha \beta} G_{6} x^{\beta},
\end{aligned}
$$

here $G_{i}(i=1,2,3,4,5,6)$ are functions of $t$ and $r=\left(x^{b} x^{b}\right)^{1 / 2}$, and the zeroth vector $h_{0}^{\mu}$ has the factor $i^{2}=-1$ to preserve Lorentz signature, and the tetrad of Minkowski space-time is $h_{b}^{\mu}=\operatorname{diag}\left(i, \delta_{b}^{\alpha}\right)$ where $(\mathrm{b}=1,2,3)$. Using the general coordinate transformation, we write

$$
h_{a \mu}=\frac{\partial \mathbf{Y}^{\nu^{\prime}}}{\partial \mathbf{Y}^{\mu}} h_{a \nu}
$$

where $\left\{\mathbf{Y}^{\mu}\right\}$ and $\left\{\mathbf{Y}^{\nu^{\prime}}\right\}$ are, respectively, the isotropic and Schwarzschild coordinates $(t, r, \theta, \phi)$. In the spherical, static and isotropic coordinate system

$$
\begin{aligned}
\mathbf{Y}^{1} & =r \sin \theta \cos \phi, \\
\mathbf{Y}^{2} & =r \sin \theta \sin \phi, \\
\mathbf{Y}^{3} & =r \cos \theta .
\end{aligned}
$$

Therefore, we obtain the tetrad components of $h^{a}{ }_{\mu}$ as

and the components of inverse matrix $h_{a}{ }^{\mu}$

$$
\begin{array}{r}
h^{a}{ }_{\mu}=\left(\begin{array}{cccc}
\sqrt{\Im} & 0 & 0 & 0 \\
0 & \sqrt{\frac{r}{\Xi}} s \theta c \phi & r c \theta c \phi & -r s \theta s \phi \\
0 & \sqrt{\frac{r}{\Xi}} s \theta s \phi & r c \theta s \phi & r s \theta c \phi \\
0 & \sqrt{\frac{r}{\Xi}} c \theta & -r s \theta & 0
\end{array}\right), \\
\text { inverse matrix } h_{a}{ }^{\mu}
\end{array}
$$

$$
h_{a}{ }^{\mu}=\left(\begin{array}{cccc}
\frac{1}{\sqrt{\Im}} & 0 & 0 & 0 \\
0 & \sqrt{\frac{\Xi}{r}} s \theta c \phi & \frac{1}{r} c \theta c \phi & -\frac{s \phi}{r s \theta} \\
0 & \sqrt{\frac{\Xi}{r}} s \theta s \phi & \frac{1}{r} c \theta s \phi & \frac{c \phi}{r s \theta} \\
0 & \sqrt{\frac{\Xi}{r}} c \theta & -\frac{1}{r} s \theta & 0
\end{array}\right) .
$$

Here, we have introduced the following notation: $s \theta=\sin \theta, c \theta=\cos \theta, s \phi=\sin \phi$ and $c \phi=\cos \phi$. After making the required calculations [41, 42], we obtain the required Møller's super-potential of $M_{\mu}^{\nu \beta}$ as given below.

$$
M_{0}^{01}(t, r, \theta)=\frac{r^{2}}{\kappa} \Im^{\prime}(r) \sin \theta \sqrt{\frac{\Xi(r)}{r \Im(r)}},
$$


and the momentum densities are

$$
\Xi_{1}^{0}=\Xi_{2}^{0}=\Xi_{3}^{0}=0
$$

Hence, one can easily find that the energy and momentum components are given by the following expressions.

$$
\begin{gathered}
E_{(\text {Moller })}^{T P}(r)=\frac{r^{2}}{2} \Im^{\prime}(r) \sqrt{\frac{\Xi(r)}{r \Im(r)}}, \\
P_{1}^{(\text {Moller })}=P_{2}^{(\text {Moller })}=P_{3}^{(\text {Moller })}=0 .
\end{gathered}
$$

This is the same energy and momentum expressions as obtained in general relativity by using the Møller energy-momentum complex. It is also independent of the teleparallel dimensionless coupling constant, which means that it is valid not only in teleparallel equivalent of general relativity but also in any teleparallel model.

\section{Examples}

In this section, we focus on some special black hole models [10] to evaluate energy and momentum distribution (due to matter and fields including gravitation) associated with them.

a) First, we consider the following form of unknown functions $\Im(r)$ and $\Xi(r)$ :

$$
\Im(r)=1-\frac{2 m}{r}, \quad \Xi(r)=\frac{(r-2 m)\left(r-\lambda_{0}\right)}{r-\frac{3 m}{2}}
$$

where $m=$ constant $>0$ and $\lambda_{0}$ is an integration constant. This solution is the vacuum case $R \equiv 0$. Thus, the line-element takes the following form;

$$
d s^{2}=\left(1-\frac{2 m}{r}\right) d t^{2}-\frac{\left(1-\frac{3 m}{2 r}\right)}{\left(1-\frac{2 m}{r}\right)\left(1-\frac{\lambda_{0}}{r}\right)} d r^{2}-r^{2}\left(d \theta^{2}+\sin ^{2} \theta d \phi^{2}\right) .
$$

The Schwarzschild metric is restored in the special case $\lambda_{0}=3 \mathrm{~m} / 2$. The metric given above was obtained by Casadio, Fabbri and Mazzacurati [43] in search for new braneworld black holes and by Germani and Maartens [44] as a possible external metric of a homogeneous star on the brane. In case $\lambda_{0}>2 m$ the metric (4.2) describes a symmetric traversable wormhole [45]. Next, In case $\lambda_{0}<2 m$, as in the schwarzschild metric, $\lambda_{0}=2 m$ is a simple horizon, and the spacetime structure depends on the sign of $\aleph=\lambda_{0}-3 m / 2$. If $\aleph<0$, the structure is that of a Schwarzschild black hole, but the space-like curvature singularity is located at $\lambda_{0}=3 \mathrm{~m} / 2$ instead of $\lambda_{0}=0$. If $\aleph>0$, the solution describes a non-singular black hole with a wormhole throat at $r=\lambda_{0}$ inside the horizon, or, more precisely, it is the minimum value of $r$ at which the model bounces. The corresponding global structure is that of a non-extremal Kerr black hole [43]. 
In this case, we obtain the following energy expressions:

$$
\begin{aligned}
E_{(\text {Einstein })}(r) & =\frac{1}{2}\left[\frac{m\left(r-4 \lambda_{0}\right)+2 r \lambda_{0}}{(4 r-6 m)^{1 / 2}\left(r-\lambda_{0}\right)^{1 / 2}}\right] \\
E_{(\text {Moller })}(r) & =E_{(\text {Moller })}^{T P}(r)=m\left[\frac{r-\lambda_{0}}{r-\frac{3 m}{2}}\right]^{1 / 2}
\end{aligned}
$$

b) Second, we choose

$$
\Im(r)=1-\frac{h^{2}}{r^{2}}, \quad \Xi(r)=r\left(1-\frac{h^{2}}{r^{2}}\right)\left(1+\frac{\chi-h}{\sqrt{2 r^{2}-h^{2}}}\right)
$$

where $h=$ constant $>0$. This form of the black hole model represents a metric with zero Schwarzschild mass. The sphere $r=h$ is a simple horizon if $\chi>0$ and a double horizon if $\chi=0$. In case $\chi<0$, the function $\Omega(r)=r^{-1} \Xi(r)$ has a simple zero at $r=r^{\prime}>h$ given by $2 r^{\prime 2}=h^{2}+(h-\chi)^{2}$ which is a symmetric wormhole throat [45]. In case $\chi=0, r=h$ is a double horizon, but a time-like singularity $\Omega \rightarrow \infty$ takes place at $r=h / \sqrt{2}$. Next, in case $0<\chi<h$, inside the simple horizon, the function $\Omega(r)$ turns to zero at $r=r^{\prime}$ which is now between $h$ and $h / \sqrt{2}$, and we obtain a Kerr-like regular black hole structure. The value $\chi=h$ leads to the simplest metric, which may be identified as a Reissner-Nordsöm black hole with zero mass and pure imaginary charge. The spacetime causal structure is Schwarzschild, with a horizon at $r=h$ and a singularity at $r=0$. In case $\chi>0$, the casual structure is again Schwarzschild but the singularity due to $\Omega(r) \rightarrow \infty$ occurs at $r=h / \sqrt{2}$.

The present example of zero mass black hole shows that, in the brane-world context, a black hole may exist (at least a solution to the gravitational equation on the brane) without matter and without mass, solely as the tidal effect from the bulk gravity.

Energy expressions associated with this case are computed exactly as

$$
\begin{gathered}
E_{(\text {Einstein })}(r)=\frac{1}{2} \sqrt{\frac{r}{1+\frac{\chi-h}{\sqrt{2 r^{2}-h^{2}}}}}\left[r-\left(1-\frac{h^{2}}{r^{2}}\right)\left(1+\frac{\chi-h}{\sqrt{2 r^{2}-h^{2}}}\right)\right] \\
E_{(\text {Moller })}(r)=E_{(\text {Moller })}^{T P}(r)=\frac{h^{2}}{r^{3 / 2}}\left[1+\frac{\chi-h}{\sqrt{2 r^{2}-h^{2}}}\right]^{1 / 2} .
\end{gathered}
$$

c) As the third example, lets consider the following form of the functions $\Im(r)$ and $\Xi(r)$ :

$$
\Im(r)=\left(1-\frac{2 m}{r}\right)^{2}, \quad \Xi(r)=\frac{1}{2}\left(r-\lambda_{1}\right)\left(r-\lambda_{2}\right)
$$

where $m=$ constant $>0$ and

$$
\lambda_{2}=\frac{m \lambda_{1}}{\lambda_{1}-m} .
$$

This is the extremal Reissner-Nordsöm black hole form, and the metric can be written as

$$
d s^{2}=\left(1-\frac{2 m}{r}\right)^{2} d t^{2}-\left(1-\frac{\lambda_{1}}{r}\right)^{-1}\left(1-\frac{\lambda_{2}}{r}\right)^{-1} d r^{2}-r^{2}\left(d \theta^{2}+\sin ^{2} \theta d \phi^{2}\right) .
$$


We obtain a black hole solution in the only case $r_{0}=r_{1}=2 \mathrm{~m}$. It is the extremal Reissner-Nordsöm metric and accordingly the effective stress energy tensor is $\widehat{T}_{\mu}^{\nu} \propto$ $r^{-4} \operatorname{diag}(1,1,-1,-1)$. Other values of $r_{0}$ lead either to wormholes (the throat is located at $r=r_{0}$ if $r_{0}>2 m$ or at $r=r_{1}>2 m$ in case $2 m>r_{0}>m$ ), or to a naked singularity located at $r=2 m$ (when $r_{0}<m$ ) as is confirmed by calculating the Kretschmann scalar 45].

When we consider this case to obtain an exact form for the general energy distribution obtained in sections 2 and 3, we find easily

$$
\begin{aligned}
E_{(\text {Einstein })}(r) & =\frac{(2 m-r)\left[r^{2}+\lambda_{1} \lambda_{2}-r\left(2+\lambda_{1}+\lambda_{2}\right)\right]}{2\left[2 r\left(r-\lambda_{1}\right)\left(r-\lambda_{2}\right)\right]^{1 / 2}} \\
E_{(\text {Moller })}(r) & =E_{(\text {Moller })}^{T P}(r)=m\left[\frac{2}{r}\left(r-\lambda_{1}\right)\left(r-\lambda_{2}\right)\right]^{1 / 2}
\end{aligned}
$$

d) The fourth example can be the following case:
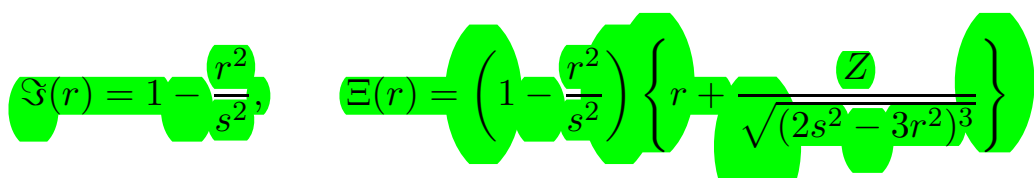

$(4.13)$

where $s=$ constant $>0$ and $Z$ is an integration constant such that $Z=0$ corresponds to integration from $r=\widetilde{r}=a \sqrt{2 / 3}$ to $r$. The value $r=\widetilde{r}$ is the one where $4 \Im(r)+$ $\partial_{r} \Im(r)$ vanishes.

The above three examples described vacuum asymptotically flat black holes solutions. Now, considering the de Sitter form of the function $\Im(r)$, we can write a solution for a vacuum configuration with a cosmological term, so that $R=4 \Lambda_{4}=12 / \mathrm{s}^{2}$, in the region $r<a$.

For this example of the Brane-World black holes one can obtain the energy relations in general relativity and teleparallel gravity as

$$
\begin{aligned}
& E_{(\text {Einstein })}(r)=\frac{1}{2} \sqrt{\frac{r}{r+\frac{Z}{\left(2 s^{2}-3 r^{2}\right)^{3 / 2}}}}\left[r-\left(1-\frac{r^{2}}{s^{2}}\right)\left(r+\frac{Z}{\left(2 s^{2}-3 r^{2}\right)^{3 / 2}}\right)\right] \\
& E_{(\text {Moller })}(r)=E_{(\text {Moller })}^{T P}(r)=-\frac{r^{5 / 2}}{s^{2}}\left[r+\frac{Z}{\left(2 s^{2}-3 r^{2}\right)^{3 / 2}}\right]^{1 / 2}
\end{aligned}
$$

e) In the last example, we have

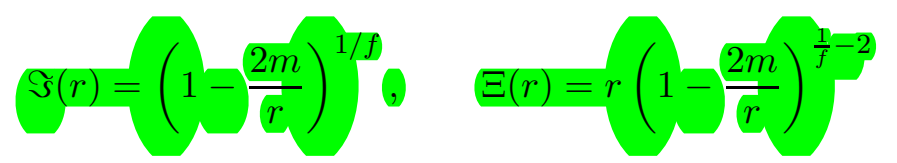

where $m=$ constant $>0$ and $f \in N$. We here try to give an example for a metric behaving non-analytically at $r=r_{h}$. The corresponding line-element is

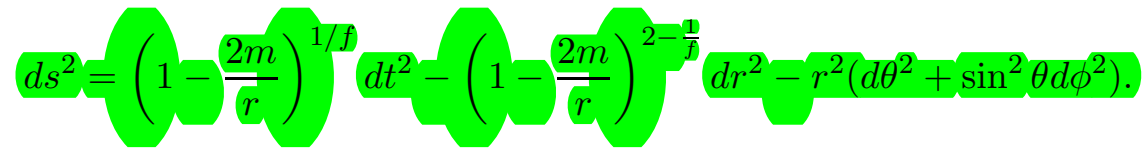




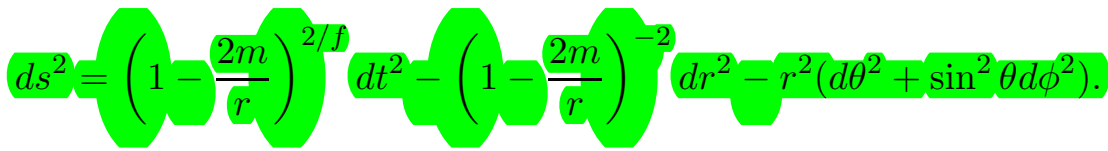

with $m>0$ and this metric $f \in N$ reveals a double horizon at $r=2 m$.

For this last example of the Brane-World black holes, we get the following expressions for the Einstein and Møller energy distributions

$$
\begin{aligned}
E_{(\text {Einstein })}(r) & =\frac{\left(1-\frac{2 m}{r}\right)^{1 / f} r^{2}-(r-2 m)^{2}}{4 m-2 r} \\
E_{(\text {Moller })}(r) & =E_{(\text {Moller })}^{T P}(r)=\frac{m}{f}\left(1-\frac{2 m}{r}\right)^{\frac{1}{f}-2} .
\end{aligned}
$$

\section{Discussions}

In the present work, in order to compute the energy and momentum distributions (due to matter and fields including gravity) associated with five different Brane-World black holes, we focus on the Einstein and Møller energy-momentum complexes in general relativity and the teleparallel gravity version of the Møller prescription.

We find that momentum components associated with Brane-World black hole models in three of the methods of calculating energy-momentum distribution of the universe are equal to zero; however, for the energy distributions, we obtain the following relations

$$
\begin{aligned}
E_{(\text {Einstein })}(r) & =\frac{1}{2} \sqrt{\frac{r \Im}{\Xi}}(r-\Xi) \\
E_{(\text {Moller })}(r) & =E_{(\text {Moller })}^{T P}(r)=\frac{r^{3 / 2}}{2} \Im^{\prime}(r) \sqrt{\frac{\Xi(r)}{\Im(r)}} .
\end{aligned}
$$

Using the above general expressions, we evaluate the energy-momentum distribution of the five different black hole models which are special cases of the general Brane-World ones.

From eqs.(70-71), one can easily see that the results obtained in teleparallel gravity are also independent of the teleparallel dimensionless coupling parameter, which means that it is valid not only in the teleparallel equivalent of general relativity, but also in any teleparallel model.

Furthermore, we show that the Møller energy distribution both in Einstein's theory of general relativity and teleparallel gravity is the same, and this interesting energy result supports the viewpoint of Lessner that the Møller energy-momentum complex is a powerful concept of energy and momentum. Our results also (a) sustains the importance of the energy-momentum definitions in the evaluation of the energy distribution of a given spacetime, and (b) supports the hypothesis by Cooperstock that the energy is confined to the region of non-vanishing energy-momentum tensor of matter and all non-gravitational fields. 


\section{Acknowledgments}

The work of M.S. was supported by the Turkish Scientific and Technological Research Council (TUBITAK).

\section{References}

[1] L. Randall and R. Sundrum, Phys. Rev .Lett. 83 (1999) 3370.

[2] L. Randall and R. Sundrum, Phys. Rev .Lett. 83 (1999) 4690.

[3] P. Hořava and E. Witten, Heterotic and type-I string dynamics from eleven dimensions, Nucl. Phys. B 460 (1996) 506 hep-th/9510209.

[4] P. Hořava and E. Witten, Eleven-dimensional supergravity on a manifold with boundary, Nucl. Phys. B 475 (1996) 94 hep-th/9603142.

[5] A. Campos, R. Maartens, D. Matravers and C.F. Sopuerta, Braneworld cosmological models with anisotropy, Phys. Rev. D 68 (2003) 103520 hep-th/0308158.

[6] P. Binetruy, C. Deffayet and D. Langlois, Non-conventional cosmology from a brane-universe, Nucl. Phys. B 565 (2000) 269 hep-th/9905012.

[7] C. Csáki, M. Graesser, C.F. Kolda and J. Terning, Cosmology of one extra dimension with localized gravity, Phys. Lett. B 462 (1999) 34 hep-ph/9906513.

[8] A. Campos and C.F. Sopuerta, Evolution of cosmological models in the brane-world scenario, Phys. Rev. D 63 (2001) 104012 hep-th/0101060.

[9] T. Shiromizu, K. Maeda and M. Sasaki, Phys. Rev. D 62 (2000) 24012.

[10] K.A. Bronnikov, V.N. Melnikov and H. Dehnen, On a general class of brane-world black holes, Phys. Rev. D 68 (2003) 024025 gr-qc/0304068.

[11] K.A. Bronnikov, G. Clement, C.P. Constantinidis and J.C. Fabris, Structure and stability of cold scalar-tensor black holes, Phys. Lett. A 243 (1998) 121 gr-qc/9801050; Grav. Cos. 4 (1998) 128.

[12] C.W. Misner, K.S. Thorne and J.A. Wheeler, Gravitation W.H. Freeman and Co., NY, 1973, p. 603 ;

F.I. Cooperstock and R.S. Sarracino, J. Phys. A 11 (1978) 877;

S. Chandrasekhar and V. Ferrari, Proc. R. Soc. London A 435 (1991) 645.

[13] A. Einstein, Sitzungsber, Preus. Akad. Wiss. Berlin (Math. Phys.) 47 (1915) 778, Addendum-ibid. 47 (1915) 799.

[14] L.D. Landau and E.M. Lifshitz, The Classical theory of fields, Pergamon Press, 4th Edition, Oxford, 2002;

P.G. Bergmann and R. Thomson, Phys. Rev. 89 (1953) 400;

S. Weinberg, Gravitation and cosmology: principle and applications of general theory of relativity, John Wiley and Sons, Inc., New York, 1972;

A. Papapetrou, Proc. R. Irish. Acad. A 52 (1948) 11;

C. Coller, On the localization of the energy of a physical system in the general theory of relativity, Ann. Phys. (NY) 4 (1958) 347;

R.C. Tolman, Relativity, thermodinamics and cosmology, Oxford Univ. Pres. London, 1934, p. 227 ;

A. Qadir and M. Sharif, Phys. Lett. A 167 (1992) 331. 
[15] G. Lessner, Gen. Relativ. Gravit. 28 (1996) 527.

[16] T. Vargas, Gen. Rel. Gravit. 36 (2004) 1255.

[17] G.G.L. Nashed, General spherically symmetric non singular black hole solutions in teleparallel theory of gravitation, Phys. Rev. D 66 (2002) 064015 [gr-qc/0212018].

[18] F.I. Mikhail, M.I. Wanas, A. Hindawi and E.I. Lashin, Int. J. Theor. Phys. 32 (1993) 1627.

[19] M. Saltı, Astrophys. Space Sci. 299 (2005) 159; Different approaches for moller's energy in the kasner-type space-time, Mod. Phys. Lett. A 20 (2005) 2175 gr-qc/0505078;

Czech. J. Phys. 56 (2006) 177.

[20] O. Aydogdu, Energy distribution of the universe in the bianchi type-II cosmological models, Fortschr. Phys. 54 (2006) 246 gr-qc/0602070; Int. J. Mod. Phys. D 15 (2006) 459.

[21] M. Salti and A. Havare, Energy-momentum in viscous kasner-type universe in bergmann-thomson formulations, Int. J. Mod. Phys. A 20 (2005) 2169 gr-qc/0502060;

A. Havare, M. Korunur and M. Salt1, Astrophys. Space Sci. 301 (2006) 43;

O. Aydogdu and M. Salti, Energy density associated with the Bianchi type-II space-time, Prog. Theor. Phys. 115 (2006) 63 gr-qc/0601133.

[22] J.M.M. Senovilla, Super-energy tensors, Class. and Quant. Grav. 17 (2000) 2799 gr-qc/9906087.

[23] L. Bel, C.R. Acad. Sci. Paris 247 (1958) 1094; Ph.D. Thesis (CDU et SEDES Paris 5e) (1960); Commun. Math. Phys. 138 (1962) 59.

[24] I. Robinson, Class. Quant. Grav. 14 (1997) A331.

[25] M.A.G. Bonilla and J.M.M. Senovilla, Phys. Rev. Lett. 11 (1997) 783; Gen. Rel. Grav. 29 (1997) 91;

J. Garecki, Annalen der Phys. 10 (2001) 911;

R. Lazkoz, J.M.M. Senovilla and R. Vera, Conserved superenergy currents, Class. and Quant. Grav. 20 (2003) 4135 gr-qc/0302101.

[26] K.S. Virbhadra, Energy associated with a kerr-newman black hole, Phys. Rev. D 41 (1990) 1086; Angular momentum distribution in kerr-newman space-time, Phys. Rev. D 42 (1990) 1066; Energy distribution in kerr-Newman space-time in Einstein's as well as moller's prescriptions, Phys. Rev. D 42 (1990) 2919; Pramana-J. Phys. 38 (1992) 31;

Pramana-J. Phys. 44 (1995) 317, Pramana-J. Phys. 45 (1995) 215; Mathematics Today 9 (1991) 39.

[27] N. Rosen and K.S. Virbhadra, Gen. Rel. Grav. 25 (1993) 429; Pramana-J. Phys. 45 (1995) 215 ;

K.S. Virbhadra and J.C. Parikh, Gravitational energy of a stringy charged black hole, Phys. Lett. B 317 (1993) 312; A conformal scalar dyon black hole solution, Phys. Lett. B 331 (1994) 302 hep-th/9407121;

J. M. Aguirregabiria, A. Chamorro, and K.S Virbhadra, Gen. Rel. Grav. 28 (1996) 1393.

[28] E.C. Vagenas, Energy distribution in $2 d$ stringy black hole backgrounds, Int. J. Mod. Phys. A 18 (2003) 5781 gr-qc/0303009; Energy and momentum distributions of a (2+1)-dimensional black hole background, Int. J. Mod. Phys. A 18 (2003) 5949 hep-th/0307162]; Effective mass of a radiating charged particle in Einstein's universe, Mod. Phys. Lett. A 19 (2004) 213 hep-th/0311050; Int. J. Mod. Phys. D 14 (2005) 573 Energy distribution in the dyadosphere of a reissner-nordstrom black hole in moller's prescription, Mod. Phys. Lett. A 21 (2006) 1947 gr-qc/0602107. 
[29] I. Radinschi, Fizika B 9 (2000) 43;

Fizika B 14 (2005) 3;

Chin. J. Phys. 42 (2004) 40;

I.-C. Yang and I. Radinschi, The energy of the gamma metric in the møller prescription, Mod. Phys. Lett. A 17 (2002) 1159 gr-qc/0110058;

I. Radinschi, Energy distribution of a charged regular black hole, Mod. Phys. Lett. A 16 (2001) 673 gr-qc/0011066;

Mod. Phys. Lett. A. 15 (2000) 803;

U.V.T., Physics Series 42 (2001) 11;

Acta Phys. Slov. 49 (1999) 789;

Acta Phys. Slov. 50 (2000) 609;

T. Grammenos and I. Radinschi, Energy distribution in a schwarzschild-like spacetime, gr-qc/0602105;

I-Ching Yang and I. Radinschi, Chin. J. Phys. 41 (2003) 326.

[30] S.S. Xulu, Møller energy for the Kerr-Newman metric, Mod. Phys. Lett. A 15 (2000) 1511 gr-qc/0010062; Energy distribution in melvin's magnetic universe, Int. J. Mod. Phys. A 15 (2000) 4849 [gr-qc/9912093]; Energy associated with schwarzschild black hole in a magnetic universe, Int. J. Mod. Phys. A 15 (2000) 2979 gr-qc/9902022]; Int. J. Theor. Phys. 39 (2000) 1153; Tolman's energy of a stringy charged black hole, Int. J. Theor. Phys. 37 (1998) 1773 gr-qc/9712100; Int. J. Mod. Phys. D 7 (1998) 773.

[31] T.T. Bringley, Energy and momentum of a stationary beam of light, Mod. Phys. Lett. A 17 (2002) 157 gr-qc/0204006;

M. Sharif and T. Fatima, Energy-momentum distribution: a crucial problem in general relativity, Int. J. Mod. Phys. A 20 (2005) 4309 [gr-qc/0410004];

M. Sharif, Energy of a regular black hole, Nuovo Cim. B19 (2004) 463 hep-th/0310151; Int. J. Mod. Phys. D 13 (2004) 1019;

T. Grammenos, Energy and momentum distributions of the magnetic solution to $(2+1)$

Einstein-Maxwell gravity, Mod. Phys. Lett. A 20 (2005) 1741 gr-qc/0412120;

R.M. Gad, Energy and momentum associated with a static axially symmetric vacuum space-time, Mod. Phys. Lett. A 19 (2004) 1847 gr-qc/0403097;

Astrophys. Space Sci. 295 (2005) 451; Astrophys. Space Sci. 293 (2004) 453;

O. Patashnick, Int. J. Mod. Phys. D 14 (2005) 1607;

P. Halpern, Energy distribution of black plane solutions, Mod. Phys. Lett. A 21 (2006) 495;

Energy of the taub cosmological solution, gr-qc/0606095;

I-Ching Yang, Chin. J. Phys. 38 (2000) 1040;

I.-C. Yang and C.-C. Jeng, On the energy of vaidya space-time, gr-qc/0605145;

I.-C. Yang and C.-H. Tsai, The evaluation of the møller energy complex in difference coordinate representations, gr-qc/0503118.

[32] M. Salti and A. Havare, Energy-momentum in viscous kasner-type universe in bergmann-thomson formulations, Int. J. Mod. Phys. A 20 (2005) 2169 gr-qc/0502060; M. Saltı and O. Aydogdu, Found. Phys. Lett. 19 (2006) 269;

O. Aydogdu and M. Salti, Energy density associated with the Bianchi type-II space-time, Prog. Theor. Phys. 115 (2006) 63 gr-qc/0601133;

O. Aydogdu, M. Saltı and M. Korunur, Acta Phys. Slov. 55 (2005) 537;

A. Havare, M. Korunur and M. Salt1, Astrophys. Space Sci. 301 (2006) 43.

[33] J.D. Brown, J. Creighton and R.B. Mann, Temperature, energy and heat capacity of asymptotically anti-de Sitter black holes, Phys. Rev. D 50 (1994) 6394 gr-qc/9405007. 
[34] S.A. Hayward, Quasilocal gravitational energy, Phys. Rev. D 49 (1994) 831 gr-qc/9303030; S.W. Hawking and G.T. Horowitz, The gravitational hamiltonian, action, entropy and surface terms, Class. and Quant. Grav. 13 (1996) 1487 gr-qc/9501014;

J.D. Brown, S.R. Lau and J. York, James W., Energy of isolated systems at retarded times as the null limit of quasilocal energy, Phys. Rev. D 55 (1997) 1977 gr-qc/9609057;

S.-T. Yau, Adv. Theor. Math. Phys. 5 (2001) 755;

C.-C.M. Liu and S.-T. Yau, Phys. Rev. Lett. 90 (2003) 231102.

[35] F.I. Cooperstock and S.A. Richardson, in Proc. 4th Canadiand Conf. on General Relativity and Relativistic Astrophysics, World Scientific, Singapore, 1991.

[36] C. Møller, Tetrad fields and conservation laws in general relativity, Proceedings of the international scholl of physics "Enrico Fermi", C. Møller ed., Academic Press, London, 1962; Conservation laws in the tetrad theory of gravitation, Proceeding of the conference on theory of gravitation, Warszawa and Jablonna 1962, Gauthier-Villars, Paris, and PWN-Polish scientifc publishers, Warzsawa, 1964, Nordita publ. no. 136.

[37] R. Weitzenböck, Invariantten Theorie, Gronningen: Noordhoff, 1923.

[38] J.A. Schouten, Ricci calculus, 2nd ed., Springer-Verlag, London, 1954, p. 142.

[39] F.W. Hehl, J. Nitsch and P. von der Heyde, in General Relativity and Gravitation, A. Held ed., Plenum, New York, 1980.

[40] H.P. Robertson, Ann. of Math. (Princeton) 33 (1932) 496.

[41] Wolfram Research, Mathematica 5.0, 2003.

[42] TCI Software Research, Scientific Workplace 5.0, 2003.

[43] R. Casadio, A. Fabbri and L. Mazzacurati, New black holes in the brane-world?, Phys. Rev. D 65 (2002) 084040 gr-qc/0111072.

[44] C. Germani and R. Maartens, Stars in the braneworld, Phys. Rev. D 64 (2001) 124010 hep-th/0107011.

[45] K.A. Bronnikov and S.-W. Kim, Possible wormholes in a brane world, Phys. Rev. D 67 (2003) 064027 gr-qc/0212112. 